Portland State University

PDXScholar

Spring 1-1-2012

\title{
Understanding the Role of Patient Activation in the Association between Patient Socio-Economic Demographics and Patient Experience
}

Katsuya Oi

Portland State University

Follow this and additional works at: https://pdxscholar.library.pdx.edu/open_access_etds

Part of the Medicine and Health Commons

Let us know how access to this document benefits you.

Recommended Citation

Oi, Katsuya, "Understanding the Role of Patient Activation in the Association between Patient SocioEconomic Demographics and Patient Experience" (2012). Dissertations and Theses. Paper 467. https://doi.org/10.15760/etd.467

This Thesis is brought to you for free and open access. It has been accepted for inclusion in Dissertations and Theses by an authorized administrator of PDXScholar. Please contact us if we can make this document more accessible: pdxscholar@pdx.edu. 
Understanding the Role of Patient Activation in the Association between Patient Socio-Economic Demographics and Patient Experience

\author{
by \\ Katsuya Oi
}

A thesis submitted in partial fulfillment of the requirements for the degree of

Master of Science
in
Sociology

Thesis Committee:

Matthew Carlson

Lindsey Wilkinson

Hyeyoung Woo

Portland State University

(C2012 


\begin{abstract}
This study focuses on the association between patient characteristics, which include both demographic and contextual factors, and patients' experiences with health care. The pre-existing literature provides rich information about patients' various demographics related to patient experience. Despite the abundance of empirical evidence showing that patients' demographics do affect how they perceive their health care. However, there is little to no empirical knowledge explaining the significance of such factors. As the existing literature points out the need for taking into contextual factors such as patient's beliefs, attitudes, skills that are pertinent to dealing with health care, my study proposes patient activation as such a contextual factor that explains the association between patient demographics and patient experience. Findings suggest that patient activation is a strong predictor of two patient experience measures: patients' rating of doctor-patient communication and their self-reported difficulties in getting needed care. However, it is also observed that the mediating effects of patient activation vary by the two dimensions of patient experiences. Though this study demonstrates that promoting patient activation may be able to normalize how patients report the quality of doctor-patient interaction, further research is needed to address access to care issues.
\end{abstract}




\section{Table of Contents}

Abstract

Table of Contents ___ ii

Chapter1

Introduction 1

Chapter2

Literature Review 6

Chapter3

Data and Methods 30

Chapter 4

Results 43

Chapter 5

Discussion 53

List of Tables

Table1.Descriptive Statistics $61-62$

Table2.Correlation Matrix among the Variables of importance 63

Table3.Ordinal Logistic Models for Doctor-Patient Communication 64

Table4.Ordinal Logistic Models for Difficulty Getting Needed Care 65

References 66

Appendices

A. The Patient Activation Measure 71

B. Waiver of Human Subject Approval 72 


\section{Introduction}

The goals of this study are to first empirically investigate the effects of patient activation on patient experiences, and then to assess the extent to which patient activation accounts for the effects of patient characteristics on the quality of doctor-patient communication and access to care. A variable of particular interest is called patient activation, which is theoretically and empirically underexplored as to its properties. The purpose of this study is to empirically investigate the potential mediating role of patient activation in the aforementioned association. Proponents of patient activation posit that being highly active equates to being able to effectively manage one's health and health care (Hibbard et al. 2004) Expanding on their contention, my research aims to provide evidence that patient activation is not only a predictor of patient experiences, but also is a factor that can explain the effects of patient demographics on patients' experiences with health care. My thesis dedicates much of its content to theoretically and empirically justifying the use of patient activation as a mediator to the association between patient demographic characteristics and patients' experiences with health care. The application of patient activation in the attempt to improve our understanding of diverse patient experiences yields information that is vital to our health care system under the Affordable Care Act. Since the success of the legislation hinges on the integration of patients into the system of care delivery, the unexplained variance in patient experience may pose a considerable obstacle to such effort to increase the efficiency of the health care system. The current literature suffers a paucity of such knowledge. A better 
understanding of differences in ways in which patients experience their health care can be used to design a viable system of care delivery that emphasizes patient-centeredness, which will become increasingly prevalent in the United States.

\section{Background and Significance}

In the past 10 years, our health care system has gone through changes to establish a system of care delivery that is less controlling. This is partially due to the prevalence of gate keeping practices that rationed costly care in the last decade of the 20th century. The public viewed, and perhaps still does, America's health care system as a heartless bureaucratic system prioritizing cost-savings over delivering high quality care (Dranove 2000). The quality of care has changed as the health care industry has undergone a number of structural changes in response to ever-increasing medical expenditures. Marcus Welby, M.D, an American medical drama television program aired from 1969 to 1976 , exemplifies the traditional role of medical professionals in the 1960s: their medical decisions were wise and infallible (Dranove 2000). During this era, patients were not to question, let alone evaluate, their doctors. This so-called era of Marcus Welby, as Dranove (2000) cogently argues, has come to an end as the health care industry has turned more attention to patient's perceptions of care.

Since the early 1970s, many health institutions have been under tremendous pressures from payers and the regulatory bodies to lower increasing rates in medical expenditures. The Health Maintenance Organization Act was passed by Congress in 1973, and was followed by the introduction of the 
Medicare prospective payment system (i.e., putting care providers on a projected budget) about a decade later. Through these waves of restructuring and reorganizing in private and public health care, assessing the quality of care now has become imperative to measure the effectiveness of health care (Shortell, Gillies, Anderson, Erickson \& Mitchell 1996). Patients' evaluation of providers has become recognized as one of the major indicators of medical performance. In fact, it is found that early intervention and preventive care, and patient education, all of which involve close coordination between patients and providers, lead to better health outcomes and thus contribute to containing medical costs greatly (Browne et al. 2010). Given this practical concern about patients' perceptions of care, various measurement tools, such as Consumer Assessment of Healthcare Providers and Systems (CAHPS) have been implemented so that patients' evaluation of their experiences with providers and systems is properly reflected in the overall assessment of medical performance.

With the prevalence of such a survey instrument, patients also can readily compare different health plans (Cleary 1999). Patients' ratings of the quality of health care that they receive are now viewed as indicative of how well patients are integrated into the process of care delivery. Also, a copious volume of evidence provided by recent studies, many of which use the CAHPS, indicates that patient experience is far from uniform; there is tendency that some patients are likely to have poorer experiences with health systems and providers (Morales, Elliott, Weech-Maldonado, Spritzer \& Hays 2001 ; 89). Given that emerging care models are attempting to implement payment mechanisms for 
providers that reflect the quality of care reported by patients through such survey tools (Chernew, Mechanic, Landon, \& Safran 2011), understanding diverse patient experiences has become more important (Browne, Roseman, Shaller, \& Levitan 2010). The recent development of the Patient Activation Measure (PAM) and its implementation to national scale surveys lends researchers a significant tool to competently address this sociological inquiry. Previous studies show that race/ethnicity and socio-economic status are markers of one's social position that either facilitates or hinders one's active involvement in their health and health care. Social scientists from different disciplines attempt to understand health disparities in our society, and there has traditionally been a strong emphasis on structural and contextual factors in health research as opposed to individual actors. Race/ethnicity, socio-economic status, and other patients' characteristics indicate the structure that individuals are placed within; yet, existing evidence does not show how individuals' beliefs, attitudes, and perceptions of their own abilities are directly related to the outcomes of research interest. A survey tool such as the PAM, on the other hand, allows us to examine such information.

Taking into account both demographics and contextual factors allows researchers to delve into inextricable dynamics between agent and structure that shapes patients' health care experiences. The following section discusses known important demographic factors of patient experiences and also the effects of patient activation on patient behavior and interactions with the health care system in general. By engaging Bourdieu's cultural capital theory, I attempt to synthesize findings from different studies into testable hypotheses. My theoretical 
framework thus lends itself to conceptually combining what we already know about race/ethnicity, socio-economic status, and patient activation in terms of patient experience. Thus, in short, by employing the PAM, this study aims to test the theory suggesting that patient activation is a link between patients' socioeconomic demographics and diverse patient experiences. 


\section{Literature Review}

In this section, I attempt to accomplish the following objectives: 1) illustrate the background in which patients' rating of care and doctors has become more salient and consequential; 2) discuss the implications of diversity in patient experience in the United States; 3 ) review studies examining the effects of patient activation on patients- namely, in what way highly activated patients are different from those who are less activated; 4) review the current literature to discuss other factors related to patient experiences. I conclude this section by making a connection between patient activation and other indicators of patient experience, leading the readers to the introduction of my theoretical framework.

Increased Importance of Patient Experience

Antagonizing both providers and patients, the public backlash against the gate keeping practice of rationing costly care had been fomented toward the end of the 21st century (Dranove 2000). The public's concerns about alienating health care seem to have gained legitimacy, as the Institution of Medicine published the book titled Crossing the Quality Chasm in 2001, reporting patient-centeredness as one of six major objectives for the U.S health system. In order to establish an effective monitoring system, it has become imperative to develop and implement reliable survey questionnaires. The Consumer Assessment of Health Providers and Systems (CAHPS), for instance, is one of the most frequently used survey tools devised for such purpose, and is now considered "the current gold standard 
for capturing patients' assessment of ambulatory care" (Morales, Elliott, WeechMaldonado, Spritzer \& Hays $2001 ; 89)$.

With the implementation of the CAHPS surveys, there have been a number of studies investigating correlates of patient experience measures. Many of their findings corroborate the salience of patients' ratings and succeed in dispelling the skepticism that patients' perceptions of care do not accurately reflect medical performance. Based on their review of existing studies, Browne, Roseman, Shaller, and Levitan (2010) surmise that patient experience is often found correlated with important health related-behaviors, such as utilization of services, adherence with medical regimens, disenrollment from health plans and providers, and the initiation of malpractice litigation. These findings are strong evidence to support the theoretical claim that the improvement of patients' experience can yield tangible benefits for our health care system.

A new care delivery system called an Accountable Care Organization, which systematically incorporates the concept of patients' evaluation of care and patient-centeredness, was proposed with the enactment of Affordable Care Act in 2010. This piece of legislation is intended to implement a less imposing and more "grass roots" approach while retaining cost-containing capabilities. Accountable care organizations, in theory, are provider-led organizations responsible for providing the full continuum of care for patients in its assigned community. The payer determines objectives for an individual $A C O$, which include setting a global budget and quality standards. Each ACO reimburses its affiliated providers on a 
fee-for-service basis. However, unlike traditional fee-for-service payment mechanisms, ACO providers are given monetary incentives to contain medical costs. That is, an ACO distributes global savings when actual spending goes blow a projected budget. In addition, although enrollees covered by this health plan are encouraged to choose recommended care providers, they are allowed to receive the same coverage and benefits regardless of their choice for care providers. Rather than "penalizing" the patients who see 'outside' providers by reducing coverage and benefits, the dissemination of necessary information and patient education are now deemed as a primary means to guide patients to make the "right" choices (Chernew, Mechanic, Landon, \& Safran 2011).

Some claim that this less intrusive approach to containing medical costs is tenable only if the continuity of care is ascertained and also complements a socalled medical home model (Liebman \& Bertko 2011: Goldsmith 2011). The concept of medical home evolved in the field of pediatrics. In 1985, for example, this care model was put to use through the Hawaii Healthy Start Home Visiting Program in response to the urgent need for the prevention of child abuse and neglect. In the following year, the programs providing medical homes for those with various disabilities were launched. Recently, increasing demand for more accessible primary care led to the proliferation of medical home programs (Goldsmith 2011). A medical home, which consists of a group of doctors, aims to provide acute, chronic, and preventive services to patients. Emphasizing accessibility and continuity, a medical home is responsible for monitoring 
assigned patients' health for a certain period of time. Theoretically, not only can it ameliorate current issues of fragmented care and limited access to care especially among Medicaid beneficiaries (Goldsmith 2009), it also attenuates patients' burden to handle information about their health on their own. In this sense, this care model nurtures close patient-doctor relationships and thus complements the Accountable Care Organization model.

This incipient care model relies heavily on effective care coordination between primary care doctors, specialists, hospitals, and patients by aligning incentives of all these parties. In short, there are mainly two things that make the care model viable: care providers' commitment to closely monitor assigned patients' health and patients' ability and willingness to work with the care providers responsible for their health. Therefore, patients who are experiencing alienation from health systems and those who are estranged from their primary care doctors may have to bear increased health risks in the near future. Furthermore, a lack of active participation of patients can severely hamper patient-centeredness and thus it may not be able to contain medical costs as efficiently as proposed. If this care model fails to demonstrate its cost-containing capability, an ACO cannot guarantee the steady distribution of global savings to its affiliated care providers. Consequently, the incentive alignment between primary physicians, specialists, hospitals, and patients is disrupted, which inevitably induces opportunism and moral hazard (e.g., selfish behaviors to hoard benefits at the cost of other parties). In this sense, it is imperative to have 
empirical knowledge suggesting the patients' characteristics that are proxies for patients' perceived estrangement from the system that they are interacting with.

\section{Patient Activation}

With established empirical and theoretical grounds, patient activation can be suggested as the variable that captures the quality of patients' relationship with the health care system in general. The careful operationalization of patient activation, the Patient Activation Measurement (PAM), also assures that patient activation is not dependent on specific medical encounters. In other words, patient activation is a patient's characteristic, not a result of prior encounters with the doctors. The PAM was developed by Judith Hibbard, Bill Mahoney and their colleagues and designed to comprehensively and objectively assess patients' level of self-advocacy, which involves the knowledge, skills, and confidence essential to managing one's own health and health care (Hibbard et al. 2004). Hibbard et al. (2004) argue that especially among chronically ill patients, activation is a key variable that would affect patients' health outcomes in the long run. They conceptualize that those who are "activated" possess the skills, knowledge, beliefs, and motivation to be active participants. With this concept, they developed a survey measurement that includes a broad array of elements that are often prerequisite to manage one's chronic illness. Coinciding with the emergence of ACOs and medical homes, addressing differences in level of patient activation at a population level has become more important. 
According to Hibbard et al (2004), a total of 18 domains were initially derived from the literature as factors that encompass patient activation. These domains were sorted into three categories: knowledge, beliefs, and skills. A panel of experts was then asked to review these domains and also a focus group of patients was conducted to verify its validity. As a result, activated patients are defined as follows; "a) those who believe patients have important roles to play in self-managing care, collaborating with providers, and maintaining their health; b) they know how to manage their condition and maintain functioning and prevent health declines; c) they have the skills and behavior repertoire to manage their condition collaborate with their health providers, maintain their health functioning, and access appropriate and high-quality care" (Hibbard et al. 2004 ; 1010).

Eighty question items were initially constructed based on this refined concept of patient activation. Hibbard et al. (2004) then conducted a pilot study with a convenience sample of 100 respondents for the further refinement of the questionnaire. Based on the data collected from this sample, Hibbard et al (2004) employed Rasch analysis to make empirically grounded decisions as to which item to be included or deleted. The following explanation of Rasch analysis is by no means comprehensive; yet, it is detailed enough to discuss the established precision and reliability of the PAM. In short, Rasch analysis allowed Hibbard et al.(2004) to provide numerical thresholds that aid them in constructing a survey tool consisting of least unbiased items (not susceptible to individual differences among patients) such as the PAM. In other words, it allows researchers to create 
a survey instrument that calibrate the difficulty of responding "agree" to an item in a way that the working of the instrument, as described below, is the same to respondents regardless of their differing characteristics. The parameter produced by such an instrument is more readily used for regression than the raw total score that often has floor and ceiling effects.

The Rasch model provides numerical thresholds to identify an item's location on the measurement scale that summarizes a person's standing along only one dimension (i.e., unidimensional) - in this case, one's level of patient activation. The process of constructing such an instrument takes two steps: developing a Rasch model and testing to see if the collected data fit the model. There are two crucial parameters produced by the Rasch model for researchers to examine throughout the process: the item locations and person locations. The proportion of "agree" responses to a given item among the sample determines the difficulty of responding "agree" to the item, which yields estimated true (unbiased) parameters. According to the calibrated measurement scale, the person locations are estimated. Subsequently, the estimated probability of responding "agree" to a given item is calculated as a logistic function of the distance between the difficulty of an item and a person's activation level. An item that yields variance too large in comparison to the true parameters is considered too discriminatory and thus subject to deletion. In this sense, it is crucial to examine standard errors associated with the person locations and item locations. Since the requirement of the Rasch model holds that the calibrated difficulty of 
the measurement should not vary by individuals (i.e., invariance of comparisons) beyond the expected values. Meeting this requirement assures the precision of the instrument. By doing so, researchers can justify their scoring - the total score means the same regardless of patients' level of activation. The data from the convenient sample of 100 respondents were needed to conduct fit tests to see which item meets the Rasch requirement and thus is justifiable to be added to the internal structure of the instrument. Again, the data do not alter the model; rather, the method of assessment (i.e., inclusion and deletion of the 80 items) should be modified to meet the requirement of invariance of comparisons as the model suggests. Also, this lends justification of my study to use patient activation as an independent variable -one's measured level of patient activation is resilient to, though not immune to, influences from prior medical encounters. In this sense, patient activation is operationalized as a patient characteristic that is quite different from patient demographics in nature. Traditionally, the existing studies are limited to studying the effects of exogenous variables (Goldstein et al.2009) on patient experience, thus there is no need to address the issues concerning causality. Patient activation is an endogenous variable in a logical sense that it is affected by the outcomes of medical encounters. However, the rigorous operationalization through Rasch analysis, which assures invariant comparisons, allows researchers to be treat patient activation disparately from patient experience. 
Hibbard et al. (2004) reported the three statistics crucial to determine which item to be included - standard error of measurement and two fit statistics indicating the reliability of the instrument predicting the distance between a person's location and a given item's location. As a result, 22 items were selected. It was also found that these selected items, taken together, measure a variable consistent with the concept of patient activation (Hibbard et al. 2004). In other words, higher participants score on this 22-item Patient Activation Measurement, the more likely they possess knowledge, skills, and beliefs necessary to effectively manage their health and health care.

Shortly after the development of the PAM, Hibbard et al (2005) set out to reduce the number of the PAM items without affecting its precision (standard error) and reliability as a whole. Hibbard et al. (2005) collected data from a random sample of 1515 adults in the United States via a telephone survey. With the increased volume of data, which affects standard errors associated with the aforementioned parameters, and iterative use of Rasch analysis, Hibbard et al. (2005) reduced the number of the PAM items from 22 to 13 without affecting the psychometric properties structured within the PAM. My study uses this short form of the PAM.

Since the development of the PAM, many studies were conducted to assess the validity of the tool. It is a topic of particular interest for health researchers to examine the association between patient activation and health utilization, especially among chronically ill individuals (Hibbard et al. 2004). Remmers et al 
(2009), in fact, found that highly activated patients are more likely to use laboratory screening tests, such as blood and lipid testing among randomly selected 1180 adults with diabetes. Similarly, a positive association between patient activation and self-management behaviors is reported by various studies. Hibbard et al (2007) conducted survey research and found that among the 479 participants, positive change in patient activation is related to positive change in a variety of self-management behaviors, such as engaging in regular exercise, asking and reading about medication side effects when taking a new prescription. Rask et al. (2009) administered the PAM to a convenience sample of 287 diabetic patients presenting to an urban public hospital diabetes clinic. They found that activated patients, i.e., those who score high on the PAM, are more likely to perform feet checks, receive eye examinations, exercise regularly, and report less difficulty in managing diabetes care.

Mosen et al (2007) collected survey data from the 4108 respondents randomly sampled among Kaiser Permanente Medical Care program members in California. They report that highly activated patients are more likely to perform self-management behaviors, use self-management services, and demonstrate high medical adherence. Furthermore, the PAM score is positively correlated with the odds of rating their overall satisfaction with the health care and services high. This finding is consistent with the results of the research conducted by Algeria et al. (2009). They examined the survey data of 1067 Hispanic respondents who were selected from a stratified, random telephone survey, and reported that - in 
addition to patients' rating of care - the level of patient activation is also strongly correlated with patients' perceived quality of doctor-patient communication. Similarly, based on the date from 2007 Health Tracking Household Survey ( $N=$ 17800), Hibbard et al (2008) conclude that highly activated patients are likely to read about possible side effects, and engage in preventative health behaviors, and have the ability to obtain needed health care services. They propose that educational approach to increasing one's level of patient activation is a key to overcoming non-financial barriers to health care. In fact, Hibbard et al. (2008) postulate that those who are competent in managing their health care and health should be able to succeed in navigating a highly complex and often confusing care delivery system. These studies consistently report the positive effects of patient activation on one's health care utilization and experiences with health care. Despite the theoretical significance of patient activation in terms of patient experience, empirical evidence to test this conjecture in the existing literature is scarce. I will describe the justifications of including patient activation as a factor explaining the association between patient demographics and patients' experiences with health care in the theory section.

\section{Other indicators of Patient Experience}

This section reviews the studies providing evidence of the relationship between patient demographics and patient experience. I conclude this section with the illustration of the gap in the literature which my study attempts to fill. Such illustration readily leads one to theoretical considerations as to the potential 
explanatory power of patient activation. Health research on patient experience mainly revolves around two kinds of explanatory variables: patient-related variables, such as race/ethnicity and socio-economic status, and provider-related variables, such as individual medical group, health plan, and care site. The review of these studies revealed that when compared to provider characteristics, patient socio-economic demographics tend to explain less variation in measures on patient experience captured by CAHPS (Goldstein 2009; Rodriguez, Scoggins; von Glahn, Zaslavsky, and Safran 2009; Weech-Maldonadoet al 2004).

Rodriguez et al. (2009) investigated how much of variation in patient's ratings of their experience with care can be explained by patients' care site, medical group, and physician among a nationally representative sample. They conduct multi-level analysis to see how much of the variance in outcomes can be attribute to different levels in our health care system. Their multi-level models include five different levels: primary care services areas (PCSA), medical groups, care sites, and physicians. Starting with PCSA, all the lower level variables are nested within those that precede them. They find that, across eight different CAHPS measures on patient experience, such as access to care and the quality of doctor-patient communication, between $27.9 \%$ to $47.7 \%$ of total variation is attributed to primary care service areas (i.e., between variance). Furthermore, individual physicians (i.e., the lowest level of aggregation) explain nearly half of the between variance especially in measures on doctor-patient communication 
and quality of chronic care. This large variability in mean outcomes implies that one's experiences with health care are highly contingent on characteristics of individual physicians and care sites.

In relation to patient characteristics, the variability of the effects of race and ethnicity on patient experiences across care providers is consistently reported, suggesting that racial/ethnic differences in patient experiences are between sites of care (Elliott et al 2009; Hasnain-Wynia et al 2007; Jha, Orav, Li, \& Epstein et al 2007). These studies suggest that non-white patients tend to receive care at a care site that delivers worse patient experiences than white counterparts. However, none of the existing studies constructs multi-level models that predict level-1 slopes (i.e., the effects of patient characteristics such as race). In other words, we do not know why the effects of minority status vary across individual care sites and individual physicians. Therefore, it is often left as conjecture as to why non-white patients tend to seek care from such providers. While these studies are useful in a sense that provider characteristics matter a great deal, under what conditions minority status are detrimental to patient experience remains unexplained.

\section{Understanding the Effects of Race/Ethnicity}

Some studies suggest that people of different race and ethnicities experience their health care differently due to the ways in which they interact with health institutions, insurance companies, and health workers (Perloff 2006). 
However, empirical evidence that can be used to test this educated conjecture is scarce in the current literature. Nevertheless, some scholars such as Lynne et al. (2010) point out that there may be more subtle and latent factors that would estrange patients from the health care system. There are many phases patients have to go through in order to receive needed care. Having health insurance only guarantees entry to the system of care delivery, though a lack of health insurance is often regarded as the primary cause of barriers to care. Insured individuals still need to initiate contact with an appropriate care site and individual care provider that can meet their needs. It is suggested that racial and ethnic diversity among patients is related to individual pathways through which patients interact with the health care system and obtain necessary care. National Healthcare Disparities Report (2009) state that Asians, Hispanics, and Blacks, when compared to Whites, are less likely to know who to turn to when they are sick, decreasing the odds of having a usual source of care by $30 \%, 40 \%$, and $10 \%$, respectively. This suggests the different level of integrations of patients into the health care system along racial/ethnic lines. Scholars such as Lynne et al. (2010) and Perloff (2006) contend that factors such as attitudes, expectations, and behaviors of the clinician and of the patient can influence the success of any clinical encounter. Their proposition implies not only that patient's values and beliefs regarding medical care are important for their health outcomes, but also that patients' congruity with expectations and institutional demands from providers is crucial determining the quality of patient experience. The evidence of disparities in patient experience even after controlling for provider-related variables stresses 
the necessity to investigate mechanisms in which patient experiences vary. Nevertheless, socio-economic status, self-rated health status, and whether or not having health insurance do not explain nearly as much of variation in patient experience as provider-related variables. All these finding point out that while patients' socio-economic demographics affect individual pathways to manage one's health and health care, those demographic variables themselves, unlike patient activation, are not direct indicators of how well patients navigate the health care system.

Yang and Kagawa-singer (2007) coin this phenomenon "patient-system cultural dissonance" and contend that cultural and linguistic diversities among patients are significant factors concerning patient experience and health outcomes. Weech-Maldonado et al. (2004) find that despite having health insurance, racial/ethnic and linguistic minorities report significantly worse experience with care when compared to native-born minorities. This difference seems clear especially among Asian respondents. While those with native fluency in English rate timeliness of care and the quality of communication with their doctors about the same as native-born Whites, the Asian respondents with limited English rate their experience worse on these measures. WeechMaldonado et al. (2004) conclude that this pattern, though not as explicit as Asians, is consistent among Hispanics as well. A similar study done by Shi, Lebrun, and Tsai (2009) finds that when adjusted for education, age, gender, and self-rated health status, those with limited language proficiency are less likely to 
have a health care visit. However, they also report that race and ethnicity remain significant in affecting patient experience. In other words, language proficiency is not the only one patient-related variable that shapes ways in which patients interact with health care systems. Some empirical studies explore the significance of language proficiency in relation to patient experiences. It is often found that patients whose primary language is not English tend to have worse access to care, report lower patient satisfaction and low quality care (Dubard \& Gizlice 2008; Hampers, Cha, \& Gutglass, et al. 1999; Vega, Karno,\& Algeria, et al. 2007). Furthermore, limited English proficiency adversely affects not only the quality of inter-personal communication with providers, but also limits patients' ability to navigate the health care system. Accessibility can be thought of as one of the elements reflecting a patient's ability to interact with the health care (Pippins, Algeria, \& Jennifer 2007). In fact, individuals with limited English proficiency are less likely to have a usual source of care (i.e., knowing who to turn to when they need medical attention). Furthermore, Pippins et al. (2007) found that even after controlling for a usual source care, limited English proficiency is correlated to the increased likelihood of reporting difficulty getting information from health workers and experiencing long waits. Quyen et al (2007) analyzed the data from a total of 2746 Chinese and Vietnamese patients receiving care at 11 health centers at 8 cities. They found that despite the use of a clinic interpreter, patients whose primary language is not English tend to give low ratings of their provider and interpersonal care. 
In relation to patient activation, one can argue that limited English proficiency may put a cap on one's level of exposure to clinical settings. As empirically observed, individuals with a limited command of English may feel estranged by the complicated processes of getting care (i.e., limited access to care, such as a lack of usual source of care) and try to avoid personal interactions with health workers that would require high proficiency in English beyond their level. In other words, in addition to language barriers, patients' language discordance can lead to more latent yet significant hindrance, such as cultural discordance (Yang and Kagawa-singer 2007). As the following section discusses in detail, patients' willingness to actively participate in their health care, which is validly and reliably captured by the PAM, can be a marker of cultural concordance between patients and the health care system. By including information obtained from the PAM, this study employs an innovative approach to understanding variance in patient experience. In addition to patients' demographics that are known as determinants of patients' relationship with the health care system, this study includes patient activation as individuals' attitudes, beliefs, and abilities related to their personal interactions with the health care system. In the following section, I discuss the theoretical salience and uniqueness of including patient activation not only as a significant determinant of the quality of patients' relationship with the health care system, but also as an explanatory variable to the association between patients' socio-economic demographics and patient experiences. 
The use of my theoretical framework is necessary for me to hypothesize the properties of patient activation in relation to patient experiences. My study is motivated by the aforementioned scholars' suggestion that a variable that accounts for personal interactions with the health care can be key to improving our understanding of vastly diverse patient experiences in the United States. Demographic variables are known to be indicators of structural advantages and disadvantages that would facilitate/hinder successful medical encounters. Yet, in terms of patient experiences, patient demographics are limited in a sense that they do not provide information of how individuals cope with or mobilize such disadvantages and advantages. Furthermore, as my literature review revealed, variability of patient demographics across different care sites and physicians suggests that the effects of patient demographics are highly contextual.

Inclusion of patient activation to this study allows me to provide empirical evidence that has been missing in the literature. In this way, we will be better informed as to exactly what patient activation accounts for in terms of variance in patient experiences. According to Hibbard et al. (2004), three major elements underlie patient activation: the knowledge, skills, and beliefs necessary to manage one's health and health care. It is patients' demonstration of such knowledge, skills, and beliefs that Shim consider as the manifestation of cultural capital. By applying the theory of cultural capital to the context of clinical settings, she proposes the concept of Cultural Health Capital (CHC). Shim (2010) surmises that the elements of $\mathrm{CHC}$ include, but are not limited to, the motivation 
to accumulate knowledge relevant to health care, and the repertoire of certain behaviors or skills that lend advantage in monitoring patients' health and health care. According to Shim (2010), these elements of CHC reflect patients' disposition to accumulate advantageous cultural products to play a "game" (Bourdieu 1990) in their favor. A game, in the context of patient activation, is to effectively manage one's health and health care, which entails self-surveillance behaviors and successful interactions with providers. Shim's theory complements our understanding of patient activation; patient activation directly assesses not only one's possession of cultural products (i.e., the abilities and knowledge to monitor one's health and health care), but also one's development of the taste and habitus to mobilize available capital (i.e., beliefs and attitudes). Furthermore, Shim's theory sensitizes us with the importance of one's deliberate mobilization of available capital, which is relatively underexplored as compared to the abundance of research on structural and demographic factors. More importantly, Shim's theory stresses the benefits of the possession of CHC on patients' personal interactions with health care. It is reasonable to reason that high activation is an indicator of CHC. Hibbard et al. (2008) concluded that highly activated patients competently navigate the health care system. Other studies also show that activated patients are more likely to adhere to medical instructions and rate the quality of doctor-patient communication higher (Algeria et al. 2009; Mosen et al 2007). 
Not only does Shim's theory augment the already grounded importance of patient activation, it also benefits this study by pointing out the inviting possibility -thatdifferences in cultural capital may reflect our hierarchical society where the unequal distribution of cultural products is evident. In other words, it can be hypothesized that there is a connection between variance in patient activation and social causes of health care disparities. The conditions or social situations under which certain cultural practices and products are valued and manifested are defined as "fields" where "players" compete (Bourdieu 1990). There is a clear divide between people who are given opportunities, incentives, and resources to develop cultural capital and those who are not. Yet, every individual plays a "game" dominated by those with advantages once they enter a given "field." Instead of being able to choose not to play such a game, those who lack cultural capital are made clear that they are dominated by more "competent" players. As a result, the disadvantaged feel alienated and discouraged to develop skills, beliefs, and attitudes to be acculturated into the given cultural context. This perceived depravation of cultural capital does not necessarily come from clinical encounters; rather it can be evoked by the sense of being alienated from the society in general. It is found that perceived everyday discrimination seems to have stronger effects on the likelihood of having necessary care than discrimination perceived during a clinic visit (Casagrande et al. 2007; Hausmann et al. 2008). It is important that patients do not feel isolated and estranged from the health care system; however, my literature review reveals that certain populations do tend to report worse patient experiences. This might be explained 
by taking into account that, as Shim (2010) suggests, people in a disadvantageous social position are deprived of incentives and opportunities to develop $\mathrm{CHC}$ due to perceived estrangement or lack of resources one way or the other.

On the other hand, those who have resources and incentives to have successful clinical encounters deliberately accumulate cultural capital by acquiring cultural products and practices, such as learning skills and knowledge pertinent to health, or properly understanding necessary information to navigate their health systems. Reaping the benefits of available capital takes one's deliberation to mobilize it to a certain goal. Individuals with the exact same demographics can have very different patient experiences without one's attitudes, beliefs, and abilities relevant to outcomes of interest taken into account. Patient activation is thus salient in a sense that it is superior to socio-economic status in predicting patient experiences.

Taking a symbolic interactionist perspective, Shim (2010) argues that individuals with cultural capital develop an identity of competent patients and hence acquire a certain disposition that influences the direction, manner, and shape of their actions (Shim 2010). In accordance with their developed disposition, competent patients both deliberately and subconsciously mobilize $\mathrm{CHC}$. It is their habitual way to maintain the psychological boundary between them (competent patients) and others (incompetent patients). Hence, " $\mathrm{CHC}$ results in the [exponential] accumulation of advantage and cultural know-how 
and thus leads to widened disparities in the quality of care delivery between those who possess CHC and those who do not" (Shim 2010: 5). As stated above, the acquisition of $\mathrm{CHC}$ is dependent on the presence of other forms of capital as well. In this regard, Shim's theory of $\mathrm{CHC}$ resonates with the "fundamental cause" perspective (Link et al. 1998; Lutfey and Feese 2005; Phelan et al. 2005) which theorizes that the distribution of resources useful in managing one's health and health care is hierarchical and reflects social inequalities and disparities in our society. In this sense, Shim (2010) illustrates a link between patient activation and social position of patients by postulating that minority or low-SES groups tend to be deprived of cultural health capital, which results in undesirable patient experiences. On the other hand, Shim also speculates that it is very possible that some racial minorities or low-SES groups possess $\mathrm{CHC}$ to offset the disadvantages of their social position; and, conversely, some non-Hispanic Whites or high-SES groups do not possess $\mathrm{CHC}$ to take advantage of their social position. In this sense, it can be suggested that what really matters in regards to patient experience is not race/ethnicity, social class, or language proficiency per se, but rather individuals' beliefs, attitudes, and abilities to offset disadvantages and utilize advantages for their goal. This theoretical conjecture is a far cry of the suggestions from the aforementioned studies (Lynne et al. 2010; Perloff 2006; Shi, Lebrun, and Tsai 2009; WeechMaldonado et al. 2004; Yang and Kagawa-singer 2007). The evidence of unexplained variance in patient experiences led these scholars to contend that there is a missing factor that needs to be taken into account among the existing 
studies. The application of Shim's CHC theory clarifies the rationale for my use of patient activation as an underexplored indicator of patient experience.

Using Shim's theory of cultural health capital is beneficial because the concept of cultural health capital provides a theoretical framework guiding me to articulate what I attempt to account for by using patient activation. Patients' beliefs, knowledge, skills encompass patient activation. It is empirically observed that patient activation is positively correlated with patient experience (Algeria et al. 2009; Hibbard et al. 2008; Mosen et al. 2007). The CHC theory complements our current knowledge of patient activation in a way that it explains why activated patients are likely to report positive experiences with the health care system. The synthesis between the theory of cultural health capital and patient activation holds that the possession of cultural capital among activated patients allows them to successfully interact with the health care system. Furthermore, low SES individuals and racial minorities are disproportionately deprived of incentives, opportunities, and resources to develop cultural capital, which is indicated by low level of patient activation among such individuals. With the PAM, I can test this hypothesis by examining to what extent patient activation explains the associations between patients' socio-economic demographics and patient experience.

Thus, the theory explaining the cause of patient activation holds that 1) patient activation is the major determinant of one's experiences with the health care; and, 2) divides along racial/ethnic, social, and economic lines reflect 
differences in the level of patient activation. As stated above, it can be hypothesized that once patient activation is taken into account, one's social position in relation to their experiences with the health care becomes significantly less important. Hence, my application of the $\mathrm{CHC}$ theory to this study, combined with the $r$ review of relevant studies, yields the following hypotheses:

1) The more activated patients are, the higher they will rate the quality of communication with their doctors.

2) The more activated patients are, the less likely they will encounter problems pertinent to access to care.

3) The effects of race/ethnicity and SES on patient ratings of care will be mediated by patient activation. 


\section{Data and Method}

This study uses data from the Health Tracking Household Survey (HTHS) conducted by the Center for Studying Health System Change in 2007. The original sample is composed of 17,797 respondents from 9,407 randomly sampled families residing in the United States. The survey was administered by telephone to households selected with a random-digit dialing method. The response rate was 43.5 percent. The $2007 \mathrm{HTHS}$ is a successor to the Community Tracking Study (CTS) Household Surveys that were conducted between 1996 and 2003 by the Center for Studying Health System Change. The 2007 HTHS provides nationally representative cross-sectional estimates of health insurance coverage, access to care, perceptions of care quality and the quality of care, the use of health services, and other topics. The 2007 HTHS is the first national survey that incorporates the questionnaire called Patient Activation Measurement (PAM), which measures patients' cultural competence. The respondents were asked whether they had one or more of 10 common chronic conditions, including diabetes, arthritis, asthma, chronic obstructive pulmonary disease, hypertension, other heart disease, cancer, skin cancer, depression or uterine bleeding. The original PAM was administered to those who reported one or more these conditions. These non-chronically ill respondents did not provide information on patient activation; thus, they were excluded. This sample screening resulted in the sample of 5,679 respondents. 
The final sample was drawn from this subpopulation after missing cases based on list-wise deletion are removed. I conducted a series of bivariate analyses to determine if those missing cases are random or related to any of the dependent variables and independent variables (confounding effects of nonrandom missing cases are most severe in such cases). Results of the analysis did not provide enough evidence to reject the null hypothesis that missing cases are random, and thus they are removed from the sample $(\mathrm{N}=5773)$. All the respondents within this sample have insurance, suffer from at least one chronic condition (self-reported), and know to whom they turn when they are in need of medical attention, i.e., they have a usual source of care. This sample screening is necessary to test the supposed effects of patient activation. Lack of health insurance and a usual source of care can be translated into a lack of exposure to the culture revolving around health care; therefore, those who report that they are activated yet do not or cannot afford to have medical encounters on a regular basis might report different patient experiences than the literature suggests.

\section{Dependent Variables}

This study uses two dependent variables representing the two dimensions of patient experience: the quality of doctor-patient communication and their report of access to care. Dependent variables are selected based on the CAHPS, which is designed to measure patient experience on various dimensions. However, the 2007 HTHS does not provide information on patient experience as extensive as the CAHPS I therefore selected patient experience measures that the CAHPS 
and the 2007 HTHS have in common. The measure for the quality of doctorpatient relationship has two aspects: communication and health promotion support, which consists of six variables from the Quality of Care Measurement (Alpha. $=.86)$. The respondents were asked how strongly they dis/agree with six statements $($ coded $0=$ strongly disagree, $1=$ disagree, $2=$ agree , and $3=$ strongly agree). Responses to the first three statements measure the quality of doctor-communication and the last three measure the level of health promotion support: 1) their doctors explain things in a way that respondents could understand, 2) their doctors treat the respondents with respect, 3) their doctors spend enough time for the respondents, 4) their doctors help them to set specific goals to improve their diet, 5) they help to set specific goals for exercise, 6) their doctors teach the respondents how to monitor their conditions.

In order to distinguish those who strongly agree with these statements from those who do not, the 6 variables are recoded into binary categories (1=strongly agree or Yes and $0=$ the other response categories or No). In this way, the fourpoint lickert scale is dichotomized into "satisfactory score" and "less than satisfactory score." I took this approach because taking the arithmetic mean of these variables results in a heavy-tailed distribution that no power transformation seems able to remedy. In fact, the majority of responses to the six question items are either 'agree' or 'strongly agree.' Therefore, recoding them into dichotomous variables makes it possible to capture the larger variation in the quality of doctor communication. Gudzune, Huizinga, and Cooper (2011) conducted a study using 
the same data and employed this dichotomization for these variables due to positively skewed response distributions. Next, I created a composite variable based on the sum of these six dichotomous variables. For the respondents who did not respond to all of the six statements, I used mean imputation by dividing the sum of the scores for the non-missing items by the number of the items to which they responded, and then multiplying the obtained value by 6 . As a result, the respondents' possible score on the quality of doctor communication ranges from 0 to 6 . Those scores of the respondents with the missing item (s) are rounded off to the nearest integer.

Access to care is operationalized as a composite variable measuring the level of difficulties getting care. This composite variable is based on two items. The first item is a question asking whether or not the respondents have ever put off their needed care for at least one of the following reasons in the last 12 months (Yes =1, and No = 0): 1) they could not use doctor of choice, 2 ) they had to wait in the office or clinic too long, 3) they couldn't get through on the telephone, 4) it took too long for them to get to the doctor's office or clinic from your house or work, 5) they could not get there when the doctor's office or clinic was open, 6) they could not get an appointment soon enough, 7) their health plan would not pay for the treatment, 8) the doctor or hospital would not accept their health insurance, and 9) they worried about the cost. The second item asks whether or not the respondents have ever been unable to get needed care in the last 12 months (Yes $=1$, and $\mathrm{No}=0$ ). I then recoded the data so that those who 
did not put off needed care for any of the reasons stated above receive 0 . In other words, those who score 1 on this measure not only had to put off their needed care but also were unable to get it at all in the last 12 months. I then took the sum of these two variables to operationalize the level of difficulties getting needed care (alpha. $=.65)$. As a result, those who score 1 on this composite measure are the respondents who had to put off needed care. Scoring 2 on this measure denotes that they had to put off needed care and were not able to get it. It is therefore intuitive to think that the level of difficulties in getting needed care is more severe for those who score 2 than those who score 1 . This operationalization of access to care is appropriate for this study since difficulties getting needed care were caused by the kind of non-financial barriers that high patient activation may be able to remedy.

\section{Mediator: Patient Activation}

The $\mathrm{CHC}$ theory proposes that various forms of capital affect cultural resonance between patients and care providers. Those include financial resources allocated for health care and cultural capital valued in the "field" of clinical settings, which Shim (2010) coins as Cultural Health Capital. An index of 13 variables from the Patient Activation Measure (PAM) was constructed (Alpha. $=.913)$ as an indicator for $\mathrm{CHC}$. This measurement consists of 13 items to which the respondents chose ne of likert scale responses (see Appendix A). Applying Rasch's analysis, it was designed in a way that a calculated score (i.e., activation score) from this measurement can be treated as an interval variable (the distance between each unit is equal in magnitude). According to Hibbard (2011), the PAM 
is a precise and accurate measure for patient activation and recently has been used by a number of studies, some of which are reviewed for this study above. This is a valid indicator for $\mathrm{CHC}$ because it measures not only the respondents' proficiency in "hard skills" necessary to navigate the health care system but also their level of confidence in doing so. That is, the higher activation score, the more likely that the respondents possess the knowledge, skills, and confidence that the culture of contemporary patient-hood expects. By the same token, the $\mathrm{CHC}$ theory proposes that those who possess ample $\mathrm{CHC}$ are likely to have those qualities and thus are likely to identify themselves as competent patients. Therefore, with this measurement, researchers can quantify one's $\mathrm{CHC}$ and thus are able to empirically observe the covariance between $\mathrm{CHC}$ and the quality of care delivery.

The respondents' activation score was calculated in accordance with the instructions given by Hibbard (2011). First, all of the responses to the 13 questions were summed to calculate a 'raw' score. For each "Strongly Disagree" response the respondent was given a 1, for each "Disagree" response given the respondent was given a 2, for each "Agree" response given the respondent was given a 3, for each "Strongly Agree" response given the respondent was given a 4. As for the respondents that did not provide the responses to all the 13 questions, the raw score was obtained by 1) dividing the sum of the scores for the non-missing items by the number of the non-missing items and 2) multiplying it by 13 . To convert the raw score into the activation score, a Rasch score table was used. With this table, an activation score from 0-100 was assigned to each 
of the calculated raw score.

Independent Variables

(Economic Hardship, Language Difficulty, Race, Education, and Income)

Five variables are operationalized as independent variables. As discussed above, this study separates patient demographics from contextual variables. Patient demographics are traditionally treated as exogenous variables, meaning that they remain constant or unchanged regardless of other variables. The use of exogenous variables is essential especially for a cross-sectional study since researchers are able to argue for the causality between exogenous variables such as race and dependent variables. However, this study extends existing knowledge by introducing patient activation to analysis as a contextual variable. To ensure the rigor of analysis, however, it is important to provide evidence supporting that patient activation is not a proxy but rather a predictor of patient experience. In order to do so, variables indicating the outcomes of patients' immediate past medical encounters should be included. Economic hardship is operationalized by creating a composite measure (alpha. $=.71$ ) consisting of five question items asking the respondents if they have had difficulties paying medical bill in the past 12 months due to 1) illness, 2) accident or injury, 3) routine health care, 4) medical test or surgical process, or 5) prescription medicines. Hence, economic hardship is a dichotomous variable; scoring 1 on 
this measure means that the respondents have had difficulties paying medical bills due to any of the reasons stated above in the last 12 months. The presence of perceived economic hardship due to medical costs indicates that they have been struggling to allocate enough financial resource to meet their medical needs. Economic hardship directly affects patients' choice of medical group, care site, doctor, and health plan, which, in turn, affects patient experience (Goldstein 2009, and Rodriguez, Scoggins, von Glahn, Zaslavsky, and Safran 2009). Furthermore, a lack of available resources may estrange patients from health care systems and thus further entrench barriers to health care (Shim 2010).

The empirical studies report that language difficulty experienced by patients is strongly associated with patient experiences with health care (WeechMaldonado et al 2004; Shi, Lebrun, and Tsai 2009). The 2007 HTHS does not provide information on patients' proficiency in English. The question item regarding language issues simply asks if they have ever had a hard time understanding what their doctors said in the past 6 months $(1=$ yes $0=$ no). Therefore, some of the reports may be irrelevant to patients' English proficiency, and rather could be due to providers' poor communication skills. This ambiguity, however, matters little in terms of analysis since I am merely interested in whether or not patients find it difficult to understand what their doctors say, not why of it.

As for race/ethnicity, dummy variables are constructed for each of the racial categories (i.e., Blacks, Hispanics, and Others), using non-Hispanic whites as a 
reference group. Education is measured by asking the respondents the number of years of education and then categorized as follows: less than high school (the number of years of education $<12)$, high school $(12)$, some college $(<16)$, having at least a four year college education (16 or more). The level of income is measured with a question asking the respondents' total annual income from all sources, before taxes and deductions (the respondents are given 10 categorical answers to choose from: $\$ 0, \$ 1-4999, \$ 5000-9999, \$ 10000-19999, \$ 20000$ 29999, \$30000-39999, \$40000-49999, \$50000-99999, \$100000-149999, and equal to or more than $\$ 1500000$ ).

\section{Control Variables}

The following three variables are included to control for their potential confounding factors: age, sex, and self-rated health status. Despite their small contribution to explained variation, all these variables are often included as "patient-mix" by many relevant studies. Age is a scale variable that ranges from 18 to over 91 (top code), and is centered at the mean (57). A dummy variable is created for female, using male as the reference group. Given the promotion of early detections for gender-specific diseases, it can be predicted that female respondents tend to utilize medical services more often and thus interact with health systems differently than men. Self-rated health status is measured with a question that asks the respondents to rate their health status, which is coded as follows; $0=$ very poor, $1=$ poor, $2=$ fair, $3=$ good to $4=$ excellent. Respondents' educational attainment and income level are coded as follows. 


\section{Analysis}

The goals of this study are to first empirically investigate the effects of patient activation on patient experiences, and then to assess the extent to which patient activation accounts for the effects of patient characteristics on the quality of doctor-patient communication and access to care. In order to assess the effects of the independent variables on the quality of doctor-patient communication, ordinal logistic regression models were esimated in SPSS. The dependent variable is ordinal; yet, the distances between each category are not necessarily equal (say, those with two "strongly agree" responses are not necessary twice as satisfied as those with one). This dependent variable thus cannot be treated as a scale variable. Ordered logistic regression yields coefficients that are more interpretable than multi-logistic regression when categories of a given dependent variable can be ranked in order. In other words, ordinal logistic regression estimates the predicted odds of choosing higher-ordered categories against choosing lower ones, which allows researchers to calculate the probability of choosing more "strongly agree" responses given a certain threshold. In this sense, the predicted values that ordinal logistic regression are more conducive to interpretations as opposed to OLS model regression which yields numerical values that were never observed (i.e., predicted values with decimal fractions). On the other hand, ordered logistic regression analysis is more constraining in a sense that it holds the effects of independent variables (slopes) constant across all the intercepts (thresholds). I address this issue by comparing the results with 
the regression coefficients estimated by conducting binary logistic regression as supplemental analysis. By running six binary logistic regression analyses estimating the odds of choosing more "strongly agree" responses against less (i.e., a) above 0 against 0 , b) above 1 against 1 and 0 , c) above 2 against 2 and less, d) above 3 against 3 or less, e) above 4 against 4 or less, and f) 6 against 5 or less), I can obtain regression coefficients comparable to those from ordered logistic regression except that all the "slopes" are free to vary.

I employ two sets of four regression models, each of which estimates the effects of independent, control, mediating variables on two different outcomes: the quality of doctor-patient communication and the level of difficulty in getting needed care. In the first model, the quality of doctor-patient communication is regressed on control variables (i.e., sex, age, self-rated health status), race, family income, and educational attainment. The estimates from the first model are compared to those from the other three models to examine 1) the effects of patient activation on the outcome after controlling for patient demographics, and 2) to what extent the regression coefficients for patient socio-demographics change after taking into account patient activation. To take into account the confounding effects of immediate past medical encounters, I add two contextual factors, asides from patient activation, to the first model: language difficulty and economic hardship. In the third model, patient activation is added to the first model instead of language difficulty and economic hardship. The differences in the estimated coefficients for patient demographics between the second and third 
mode yield the essential information to demonstrate how patient activation is different from other two contextual factors in mediating the net effects of patient demographics, which essentially addresses the second research question.

All of the variables are included in the final model. These models extend the existing studies of patient experience by estimating the unique and combined effects of patient activation and other suggested indicators. The final model yields the information vital to address both of my research questions, since its estimates allow me to make a conclusion about the explanatory and mediating, of patient activation after patients' immediate past experiences are taken into account. Based on the review of previous studies and the application of the $\mathrm{CHC}$ theory, it is expected not only that patient activation will have a significantly positive effect on the quality of doctor-patient communication, but also that when patient activation is taken into account; the other variables will cease to be statistically significant.

The second analysis of access to care is estimated using ordinal logistic regression commands in SPSS. The analysis proceeds in the same way as the analysis of the quality of doctor-patient communication since the structure of this dependent variable is the same (i.e., ordinal yet not scale). The descriptive analysis shows that this variable calibrates the difficulty in getting a higher score, and thus the use of ordinal logistic regression is justifiable. According to Hibbard et al (2008), it is suggested that activated patients are able to effectively navigate the health care system and thus less likely to encounter problems getting needed 
care. Therefore, a higher score on patient activation is associated with an increase in the odds of reporting no problems obtaining needed care. 


\section{Results}

Table 1 presents the unweighted descriptive statistics for the sample, 80 percent of which is White, $11 \%$ Black, $4 \%$ Hispanic, and $4 \%$ Others. Female respondents comprise nearly $60 \%$ of the sample. The Patient Activation Measure score, which is based on responses to the 13 items asking how active patients are in terms of managing their health and health care that they received in the past 12 months, ranges from 13.30 to 100 with a mean of 67.43 . According to the study using the entire sample (nationally representative) of 17,800 from 2007 HTHS, approximately 44 percent of the sample scored higher than 67.I (Hibbard et al. 2009). Therefore, the level of activation within this subpopulation is, on average, not particularly higher or lower than that of the entire sample. Age ranges from 18 to 91 with a mean of 58.45 . In addition, nearly 60 percent of the respondents responded that their household income exceeds $\$ 40,000$, while 22 percent of them have had problem(s) paying medical bills in the past 12 months. In terms of education, approximately 89 percent of the sample graduated from high school and 55 percent of them received some college-level education (i.e. the number of years of education is more than 12).

Nearly $46.6 \%$ of the sample responded to all the 6 items asking about the way doctors treat the respondents with less than 3 "strongly agree"s, while approximately $38.8 \%$ of the respondents in this sample report higher satisfaction with communication with their doctors. Nearly $80 \%$ of the sample reported no problems in getting needed care and $13.2 \%$ of them had to put off getting needed 
care. $7 \%$ of the respondents in this sample not only had to put off getting needed care but also have been unable to get needed care at all at least once in the past 12 months.

Table 2 presents the correlation matrix showing how strongly independent variables are related to the two outcomes and patient activation. It is clear that family income is significantly associated with all the three variables. As predicted, family income is positively associated with the level of patient activation $(r=.16)$, which means that higher family income they earn, it is more likely for them to be activated. Also there is weak positive association between the level of patient activation and education; those who do not have any college education are likely to be less activated than those who are more educated. Surprisingly, the strength of association between race and the level of activation is found almost negligible. On the other hand, table 2 shows that contextual factors capturing patients' immediate past experiences are moderately associated with the two patient experience measures and patient activation, indicating that language difficulties and economic hardship are confounding factors that need to be controlled for. For example, those who had difficulties understanding their doctors and paying medical bills are likely to be less activated and also have negative experiences compared to those who never had such experience at all in the past 12 months ( $r$ $=-.216$, and -.156 respectively). Thus, it is observed that language difficulties and economic hardship are associated with both the mediator (i.e., patient activation) and the outcomes (i.e., doctor-patient communication and access to care). 
Table 3 presents the regression coefficients estimating the effects of the independent variables on the quality of doctor-patient communication. As fully discussed above, Model 1 includes demographic independent variables: race and SES variables, and Model 2 adds the two contextual variables in order to analytically isolate the mediating and explanatory effects of patient activation from other two contextual variables (i.e., language difficulties and economic hardship), which are both theoretically and empirically found as confounding factors. In addition to the results from the bivariate analysis discussed above, this supposition was also made according to the bivariate associations between patient demographics and the two contextual variables (the results are not shown). Model 3 added patient activation to Model 1 to see the mediating effects of patient activation. Model 4 estimates the independent effect of patient activation as a covariate. All of the hypotheses are supported when the mediating and explanatory effects of patient activation are observed in Model 3 and remain significant in Model 4. All the estimates reported here are statistically significant with the confidence level above 95\%.

In Model 1, it is shown that a one unit increase in family income is associated with approximately a $5 \%$ increase in the odds of reporting the higher quality of doctor-patient communication. Consistent with the results of bivariate analyses, those with low self-rated health status are likely to report lower satisfaction with doctor-patient communication. It is also worth noting that the coefficients for all the racial variables are not statistically significant. With socio- 
economic status and various control variables taken into account, this model estimated that race does not affect how patients perceive the quality of doctorpatient relationship.

In Model 2, the negative effects of language difficulty and economic hardship on the perceived quality of doctor-patient communication are found notably significant; decreasing the odds of having higher satisfaction by approximately $51 \%$ and $20 \%$, respectively. Lastly, the estimated effects of all the racial variables remain insignificant in this model.

Model 3 added patient activation to Model 1 . The -2 log likelihood test indicates the magnitude of the effect of patient activation on the dependent variable. In fact, one standard deviation increase in PAM (16.73) increases the odds of having higher satisfaction with doctor-patient communication by approximately $220 \%$. The coefficient for self-rated health status and age in Model 3 , which are shown statistically significant in both Model 1 and 2 , are no longer significant, meaning that patient activation explains away the positive effect of having higher health status and age. Similarly, it is also found that when patient activation is taken into account, family income ceases to have a significant effect on the likelihood of choosing more "strongly agree" responses. These observed mediating effects are unique to patient activation, since the two indicators of patients' immediate past experiences do not explain why family income matters in terms of patients' perceptions of doctor-patient communication. 
Model 4 is employed to assess the mediating effects of patient activation after controlling for patients' difficulty in understanding what their doctors are saying and paying medical bills. A brief inspection reveals that the explanatory and mediating factors of patient activation are still observed in Model 4. It is also found that patient activation explains away the significance of economic hardship. When one compares the estimated coefficients in Model 4 to those in Model 2 and 3, it becomes clear that a) increases in patient activation positively affect the likelihood of reporting doctor-communication more favorably regardless of one's economic hardship and language difficulty; b) experiencing economic hardship per se does not necessary adversely affect doctor-patient communication. Also, patient activation partially mediates the negative effect of having language difficulties, which is indicated by the decrease in the magnitude of its effect when Model 2 is compared to 4 . In fact, having difficulties understanding the doctors decreased the odds of reporting higher satisfaction with doctor-patient communication by approximately $42 \%$ in Model 4 , and $51 \%$ estimated by Model 2. Across the different models, race variables are not statistically significant, indicating that in terms of doctor-patient communication, my categorization of race does not explain variance in patient experiences. The magnitude of the independent effect of patient activation is enormous; it is estimated that the probability that middle class white males (those who earn the family income of $\$ 30000$-39999 and graduate from some college) at the age of 58 with the minimum level of patient activation (13) give a "strongly agree" to all the six questions is approximately .0038 (the expected odds of giving five 
"strongly agree" responses or less $=6.249-\operatorname{EXP}\left(0.011^{\star} 5+0.067^{*} 13-0.003^{\star} 58-\right.$

$\left.0.044^{*} 3\right)$. On the other hand, the probability for the individuals with the same characteristics who are the most activated (100) is .568. This demonstrates that, with everything else equal, patient activation has a tremendous influence on how patients perceive the quality of doctor-patient communication. As further discussed in the following section, it can be concluded that patient activation does mediate some of the known effects of patient demographics on the perceived quality of doctor-patient communication, such as income. Comparison between Model 2 and 3 reveals that patient activation alone accounts for the significance of family income. Even when taken together with language difficulty and economic hardship in Model 4, the explanatory and mediating effects of patient activation persist.

I would like to remind readers that ordered logistic regression assumes that all of the estimated coefficients for variables are constant across different thresholds. To examine the plausibility of this assumption in my sample, I conducted binary logistic regression analysis for the same thresholds to see if estimated regression coefficients do not vary. Though results are not shown, it is revealed that across all the thresholds, the estimated effects of patient activation, economic hardship, and language difficulty remain relatively constant. In the final model of binary logistic regression, the odds ratios for these variables range from 1.069 to 1.074 , from .833 to 1.029 , and from .494 to .605 , respectively. All of these ranges fall into the $95 \%$ confidence intervals estimated by the ordinal 
logistic regression models. In other words, regardless of which category is used as a threshold compared to its higher-ordered counterparts, the net effects of those variables remain constant, which validates the results from my ordinal logistic regression.

Table 4 presents regression coefficients estimating the effects of the independent variables on the odds of reporting problems getting needed care (either they had to put off or could not obtain needed care). In Model 1, regardless of age, sex, and self-rated health status, being "others" (i.e., those who are not Hispanic, black, or white) increases the odds of reporting the problems by approximately $66 \%$. In terms of SES, the model indicates mixed findings. It is estimated that those whose do not have four year of college-level education are less likely to report the problems of getting needed care, compared to those who have. In contrast, the effects of family income are shown positive, meaning that the higher family income they earn, the less likely that they encounter problems getting needed care.

In Model 2, it is shown that language difficulties and economic hardship increase the likelihood of not being able to get needed care. Especially economic hardship is shown as a salient determinant of access to care problems, it nearly quadruples the odds (the odds ratio $=3.979$ ). Furthermore, positive effects of being less educated become even stronger after taking into account these two variables. The effects of not having finished high school, not having gone to college, not having a four year college education decrease the odds of having 
access to care problems by approximately $50 \%, 30 \%, 20 \%$, respectively. It is thus clear that education and the likelihood of not being able to get necessary care are positively associated. The absolute value of the coefficient for Black is increased and now significantly associated with a decrease in the odds.

Model 3 illustrates the effects of patient activation, which are found to be significant. It is found that one standard deviation increase in the level of activation leads to a decrease in the odds of reporting problems getting needed care by approximately $30 \%$ (the odds ratio $=\exp (-.022 * 16.7))$. However, patient activation does not seem to mediate the effects of patients' socio-demographics. In fact, compared to Model 1, the estimated effects of the education variables and "other" race in Model 3 remain almost unchanged. These negligible mediating effects of patient activation are also indicated by a change in the pseudo-R square showing that patient activation did not improve the overall model fit. With only patient activation taken into account, the negative effect of being "other" race remains statistically significant. While patient activation mediates the effect of family income and self-rated health status on doctorpatient communication, it does not account for the significance for any of the demographics in terms for access to care.

Model 4 estimates the extent to which patient activation mediates the effects of patients' socio-demographics while taking into account economic hardship and language difficulty. This regression model also shows, consistent with the results from Model 3, that despite the statistical significance of patient 
activation, its mediating effects on other significant independent variables are meager. . One notable change seen in Model 4 is that the net effect of family income is no longer statistically significant — the majority of its effect is, however, mediated rather by economic hardship and language difficulty, instead of patient activation alone. Furthermore, the regression coefficients show that not having any college education decreases the odds of difficulties getting needed care by at least $36 \%$. This pattern -the less educated they are the less likely that they report access to care issues is consistent across all the four regression models. Regardless of the level of activation, having high self-rated health status is beneficial in terms of getting care. Based on the estimated coefficients, the probability that least activated (13) white males with moderate income $(\$ 30,000$ 39,999 ), education (some college), and health status (fair) at the age of 58 report no problems getting care is approximately .775 (The expected odds of getting no difficulty getting needed care $=\operatorname{EXP}(-.262 * 1-0.020 * 13+-0.03 * 58-0.234 * 3-$ $\left.0.031^{*} 5\right)$ ). Furthermore, the risk of experiencing difficulties getting care is dramatically increased by economic hardship and communication problems. When everything else equal, such disadvantages can nearly halve the probability of reporting no problems (.36). On the other hand, the positive effect of patient activation can potentially overcome those disadvantages - with everything else held constant, increasing the level of patient activation from lowest (13) to highest (100) can raise the probability as high as .75 . This demonstrates that patient activation can, in magnitude, offset the effects of medical bill and language problems. However, Model 4 also shows that patient activation does 
not mediate the effects of language difficulty and economic hardship on the likelihood of encountering access to care issues. Therefore it can be suggested that highly activated patients are affected by those disadvantages in terms of getting needed care. When these results are compared to those from binary logistic regression analyses (results not shown) to assess to what extent regression coefficients vary by different thresholds. It is revealed that the coefficients estimated by the two different logistic regression analyses are not significantly different from one another. In fact, the test for parallel lines supports this claim $(\alpha>.05)$. It is clear that the effects of patient activation on different aspects of patient experience are not uniform. The following conclusion section discusses how each of the proposed hypotheses is rejected or supported in detail, as well as the implications of such findings. 


\section{Discussion}

\section{Doctor-Patient Communication}

Based on the findings discussed in the previous section, there is enough evidence to support the first proposed hypothesis: the more activated patients are, the higher they will rate the quality of communication with their doctors. Without contextual variables (i.e., language difficulty, economic hardship, and patient activation), only family income and self-rated health status were found significant; and patient activation alone explains the significant effects of family income, self-rated health status. In this sample, it is found that patients' rating of doctor-patient communication does not vary by race. This pattern is persistent across all four regression models. It is thus concluded that my study supports the third hypothesis: patient activation mediates the effects of patient demographics on the quality of doctor-patient relationship.

Hibbard (2009) originally proposed that patient activation encapsulates one's attitudes, beliefs, and skills to manage one's health and health care. The application of Shim's cultural health capital leads me to stress the most salient aspect of patient activation in terms of face-to-face medial encounters. All the cultural products and practices that patients demonstrate to their doctors are translated into a form of symbolic capital that signals patients' certain "taste" validated by the culture of clinical settings. This form of symbolic capital induces 
patients' deservedness that is perceived by their doctors. As a result, patients are able to elicit better quality communication with doctors.

The salience of perceived deservedness of patients is pointed out not only by Shim (2010), but also supported by empirical evidence. By using data from the 1988 National Survey of Family Growth, Saperstein (2010) reports that it is patients' perceived race, as opposed to their self-identified race, that affects the likelihood of eliciting health screening support from their doctors. Her study suggest that what matters in terms of face-to- face medical encounters is patients' lack of symbolic capital often associated with racial minorities. Although my study finds no racial disparities in doctor-patient communication, her study nevertheless lends empirical support that the presence or absence of one's cultural capital becomes manifest on doctor-patient communication. Furthermore, Bugyi (2009) finds that patients' level of ease and comfort with doctors determines how compliant patients are. As Shim's theory suggests, establishing doctor-patient relationship by activating patients' cultural capital leads to desirable medical encounters. Feeling that their health status is low, the presence of economic hardship, and low income earnings are structural issues that potentially keep patients from being activated (all these variables are strongly correlated with both the PAM and the rating of doctor-patient communication). Conversely, activated patients are able to overcome such structural barriers and establish a positive relationship with their care providers. 
My findings also support the second hypothesis: the more activated patients are, the less likely they will encounter problems pertinent to access to care. The estimated effects of patient activation are constant in both model 3 and 4 . Patient activation and the likelihood of reporting any difficulties in getting needed care are thus in a linear relationship; the more activated you are, the less likely that you will encounter access to care problems. While this study does not demonstrate the causality of the association between the likelihood of reporting access to care and patient activation, it still provides informative empirical knowledge: patient activation makes unique contribution to explaining variance in access to care issues regardless of race, education and income, or patients' immediate past experiences with the health care system. While this evidence is supportive to the $\mathrm{CHC}$ theory explaining why patient activation positively affects patient experience, it does not lend itself to understanding the significance of demographics in terms of access to care. Difficulties in getting needed care, in contrast to doctor-patient communication, do not necessarily involve face-to-face interactions with health workers and medical staff. It is theoretically supposed that the presence of cultural capital helps patients to effectively navigate the health care systems. Hibbard et al. (2009) also suggests that being tactful in obtaining needed care in a timely manner is one of the qualities that activated patients possess. However, the evidence presented by this study suggests that the acquisition of this skill - whether or not being able to effectively navigate the health care system to obtain necessary care is determined by one's social position (patient activation would mediate the effects of demographics 
otherwise). One therefore needs to cautiously scrutinize the claim that stratification along the line of socio-economic status reflects the unequal distribution of useful skills and abilities to effectively deal with the health care system (Bugyi 2009; Shim 2010). Though future research is necessary to investigate how those activated patients experience health care, this study presents indisputable evidence that our health care system should encourage the promotion of patient activation in the attempt to increase the level of activation at a population.

It is thus concluded that my study does not support the third hypothesis in terms of access to care. The varying degrees of mediation by different patient experience measures spark a series of interesting conjectures, some of which are worth noting. It can be suggested that access to care concerns two different yet intertwined aspects: the skills necessary to effectively navigate the health care system and the expectations/needs/wants that are shaped by ones' social position and life situations. For example, being more education leads patients to be aware of the importance of quality care. Higher expectations among educated patients result in the increased likelihood of reporting access to care issues as they are more aware of system failures than those who are less educated. This conjecture, though lacking corroborating empirical evidence, might explain the relatively smaller effect of patient activation on access to care- Increases in the level of patient activation too, by theory, lead patients to have more needs and wants. In other words, patient activation increases the likelihood of encountering 
access to care issues since activated patients are more demanding than those who are not, while facilitating them to effectively meet their needs. Access to care issues are also intertwined with factors that this study failed to take into account, mainly concerning patients' health insurance and geographic information. In other words, regardless of one's level of patient activation, the range of choices that patients can sift through is demarcated by individual insurance, the quality of which often rests on their employment. The limitations on choice imposed by health insurance are further confounded by regional characteristics. The trends in care delivery vastly vary by, for example, the prevalence of HMOs (Feldstein 2005 ) in the given region. While the need for patient centeredness is brought to federal attention, the capacity of individual practice to accommodate such need is by no means uniformly. In other words, there are limits as to how successfully individuals can secure needed care in a timely manner. I would like to remind readers the discussion of explained between/ within variance in patient experience associated between individual care providers, care sites, and systems. Such discussion is not yet informative since random effects at both individual and provider levels remain unexplained; one cannot discern when patients choose to seek care from providers or systems that deliver substandard patient experience and when they are merely "placed" regardless of their will. This study contributes to the existing knowledge with the evidence that access to care issues cannot be overcome by individuals alone, no matter how active in managing health and health care they are. 


\section{Limitations and Future Research}

The results of this study were interpreted with caution due to some limitations mostly inherent in the data set. Since my data set is cross-sectional, it is not empirically plausible to establish the causality of the associations whose time order is not logically clear. In my study, this limitation mainly concerns the effects of patient activation on the dependent variables. Some studies find that the level of patient activation is influenced by medical encounters - trust and certain practice climate that facilitates patients' active involvement may induce higher activation (Becker \& Robin 2009). Thus failing to establish causality leaves room for alternative explanation that reverses the direction of the relationship between patient activation and the quality of doctor-communication that is assumed by this study. Regardless, this alternative explanation does not entirely invalidate the conclusion that patient activation is a strong indicator of, if not a cause of, the quality of doctor-communication. The same limitation is applied to estimating the likelihood of reporting difficulties getting needed care. It may be that such poor patient experiences cause patients to report lower level of activation, or that high activation is an indicator of successful medical encounters. These limitations therefore call for future research assessing changes in patient activation and the quality of medical encounters over time.

This dataset does not contain care provider information, which is often found a more important indicator of patient experience. Not being able to control for individual care site, health plan, and care provider most likely result in large 
unexplained variance on various patient satisfaction measures (Rodriguez et al. 2009). Given the empirical evidence suggesting that providers' practice style does affect both patients' level of activation and their satisfaction, care provider information can be potentially confounding to the association between patient activation and patient experience. Therefore, the current literature can benefit from future studies investigating the effects of patient activation while taking into account within and between differences across provider-related variables, such as health plan, care site, and care provider.

Lastly, it is possible that sample screening resulted in selection issues hampering the generalizability of my sample. Since a usual source of care and having health insurance are often associated with positive patient experience, including more accessibility of care; therefore, it is likely that the respondents within my sample generally rated patient experience more favorably. While this selection issues make it problematic to generalize my findings to the population, this purposeful sample selection was necessary to address the validity of the PAM. Hibbard (2009) tested the PAM by administering it to the participants recruited through convenient sampling. Especially the sample used to calibrate the PAM scale and refine the questionnaire is recruited through newspaper advertisements and the PAM items were administered through a telephone interview (Hibbard 2004). The information was collected from the individuals that read newspaper regularly and are interested in participating in health care, which indicates their certain level of exposure to the culture of clinical setting. It can be 
inferred that the validity of the PAM may be questionable when it is administered to those who do not have or avoid medical encounters on a regular basis. Those who report chronic conditions yet do not see a doctor regularly due to a lack of the means to do so or simply aversion to health care. Therefore, those individuals are excluded from the study due to my concerns of them threatening the validity of the PAM. 
Table 1. Descriptive statistics for variables $(N=5573)$ Source:2007 HTHS

Variable Name

Race

Black

Hispanic

Others

White

Family Income

$\$ 0$,

$\$ 1-4999$

$\$ 5000-9999$,

$\$ 10000-19999$

$\$ 20000-29999$

$\$ 30000-39999$

$\$ 40000-49999$

$\$ 50000-99999$

$\$ 100000-149999$

$>\$ 1500000$

Education

Less than high school

High school

Some College

$=<$ a four year college education

Language Difficulties

Economic Hardship

Sex

Male

Female

Self-rated Health

VeryPoor

Poor

Fair

Good

Excellent
Desciption

$\%$

11.10

4.20

4.10

80.60

Self-reported annual household Income

1.30

3.00

5.80

11.70

11.10

10.40

9.30

28.30

10.80

8.20

Highest level of education attained

10.70

34.20

24.50

30.60

Have ever had difficulties

understanding what the doctors are

saying

Have ever had difficulties paying $\quad 22.50$

medical bills

49.00

Self-rated heal th status

20.00

32.50

28.90

9.20

Mean (SD) $58.4(15.5)$

the Patient Activation Measure 
Table 1 (cont.). Descriptive statistics for variables $(\mathrm{N}=5573)$ Source:2007 HTHS Variable Name Desciption

\section{Doctor-patient communication}

0

1

2

3

4

5

6

Difficulties getting care 0

1

2
The number of "strongly agree" responses to six questions

25.00

11.90

8.70

15.90

11.70

9.90

16.80

Self-reported problems when getting care No difficulties

79.90

Had to put off needed care

13.20

Had to put off AND failed to get needed

6.90 
Table2. Correlation Matrix of Analytic Variables ( $N=5573$ ) Source:2007 HTHS

\begin{tabular}{lccc}
\hline & D-P Communication & Access to Care & Patient Activation \\
B & -0.006 & 0.020 & $-0.039^{*}$ \\
H & $-0.027^{*}$ & 0.024 & -0.023 \\
O & 0.006 & $0.070^{*}$ & 0.013 \\
$\mathrm{FI}$ & $0.106^{*}$ & $-0.080^{*}$ & $0.160^{*}$ \\
LH & $-0.070^{*}$ & -0.013 & $-0.094^{*}$ \\
High & $-0.012^{*}$ & -0.005 & $-0.069^{*}$ \\
SomeC & -0.011 & 0.033 & 0.011 \\
Lan & $-0.167^{*}$ & $0.144^{*}$ & $-0.139^{*}$ \\
Econ & $-0.081^{*}$ & $0.331^{*}$ & $-0.115^{*}$ \\
Age & $-0.043^{*}$ & $-0.209^{*}$ & $-0.062^{*}$ \\
F & -0.016 & $0.099^{*}$ & 0.017 \\
SH & $0.124^{*}$ & $-0.149^{*}$ & $0.241^{*}$ \\
Doc & 1.000 & $-0.156^{*}$ & $0.537^{*}$ \\
Care & $-0.156^{*}$ & 1.000 & $-0.147^{*}$ \\
PA & $0.537^{*}$ & $-0.147^{*}$ & 1.000 \\
\hline
\end{tabular}

Note: $\quad *=\mathrm{P}<.01$

$\mathrm{B}=\mathrm{Black}, \mathrm{H}=\mathrm{Hispanic}, \mathrm{O}=$ Others, $\mathrm{Fl}=$ Family Income, $\mathrm{LH}=$ Less than High School, SomeC $=$ Some college, Lan=Language Difficulties, Econ=Economic Hardship, $\mathrm{F}=$ Female, $\mathrm{SH}=$ Self-rated health status, Doc=Doctor-patient communication, Care $=$ Access to care, $\mathrm{PA}=$ Patient Activation 
Table 3. Ordinal Logit Regression Coefficients Estimating Effects of Patient Characteristics on the Quality of DoctorPatient Communication ( $\mathrm{N}=5573$, Source: $2007 \mathrm{HTHS}$ )

\begin{tabular}{|c|c|c|c|c|c|c|c|c|}
\hline & \multicolumn{2}{|c|}{ Model 1} & \multicolumn{2}{|c|}{ Model 2} & \multicolumn{2}{|c|}{ Model3 } & \multicolumn{2}{|c|}{ Model 4} \\
\hline & Coefficents & SE & Coefficent & SE & Coefficient & SE & Coefficient & SE \\
\hline \multicolumn{9}{|l|}{ Independent Variable } \\
\hline \multirow[t]{2}{*}{ Black } & 0.068 & 0.078 & 0.089 & 0.080 & 0.134 & 0.079 & 0.115 & 0.082 \\
\hline & 1007 & & 1093 & & 1.143 & & 1123 & \\
\hline \multirow[t]{2}{*}{ Hispanic } & -0.126 & 0.121 & -0.115 & 0.123 & -0.146 & 0.123 & -0.142 & 0.126 \\
\hline & 0.882 & & 0.891 & & 0.862 & & 0.866 & \\
\hline \multirow[t]{2}{*}{ Others } & 0.135 & 0.120 & $0.250^{*}$ & 0.124 & 0.142 & 0.123 & 0.236 & 0.127 \\
\hline & 1145 & & 1284 & & 1.153 & & 1.270 & \\
\hline \multirow[t]{2}{*}{ Family Income } & $0.046^{* *}$ & 0.049 & $0.033^{* *}$ & 0.013 & 0.020 & 0.013 & 0.011 & 0.013 \\
\hline & 1047 & & 1034 & & 1.020 & & 1014 & \\
\hline \multirow[t]{2}{*}{ Less than High School } & $-0.224^{*}$ & 0.096 & -0.186 & 0.098 & -0.104 & 0.098 & -0.088 & 0.100 \\
\hline & 0.799 & & 0.830 & & 0.901 & & 0.916 & \\
\hline \multirow[t]{2}{*}{ HighSchool } & -0.046 & 0.063 & -0.005 & 0.065 & 0.076 & 0.082 & 0.097 & 0.083 \\
\hline & 0.955 & & 0.995 & & 1.067 & & 1102 & \\
\hline \multirow[t]{2}{*}{ Some College } & -0.113 & 0.066 & -0.081 & 0.067 & -0.057 & 0.067 & -0.044 & 0.068 \\
\hline & 0.893 & & 0.922 & & 0.945 & & 1061 & \\
\hline \multirow[t]{2}{*}{ Language Difficulty } & & & $-0.712^{* *}$ & 0.068 & & & $-0.544 * *$ & 0.070 \\
\hline & & & 0.491 & & & & 0.580 & \\
\hline \multirow[t]{2}{*}{ Economic Hardship } & & & $-0.215^{* *}$ & 0.062 & & & -0.055 & 0.064 \\
\hline & & & 0.807 & & & & 0.946 & \\
\hline \multicolumn{9}{|l|}{ Mediator } \\
\hline \multirow[t]{2}{*}{ Patient Activation } & & & & & $0.068 * \cdots$ & 0.002 & $0.067^{* * *}$ & 0.002 \\
\hline & & & & & 1.070 & & 1069 & \\
\hline \multicolumn{9}{|l|}{ Control Variable } \\
\hline \multirow[t]{2}{*}{ Age } & -0.002 & 0.002 & $-0.005^{* *}$ & 0.002 & -0.002 & 0.002 & -0.003 & 0.002 \\
\hline & 0.998 & & 0.995 & & 0.998 & & 0.997 & \\
\hline \multirow[t]{2}{*}{ Female } & -0.037 & 0.049 & -0.035 & 0.050 & -0.090 & 0.050 & -0.094 & 0.051 \\
\hline & 0.964 & & 0.966 & & 0.912 & & 0.910 & \\
\hline \multirow[t]{2}{*}{ Self-Rated Health } & $0.155^{* *}$ & 0.023 & $0.118^{* * *}$ & 0.024 & -0.028 & 0.024 & -0.044 & 0.025 \\
\hline & 1168 & & 1125 & & 0.972 & & 0.957 & \\
\hline \multicolumn{9}{|l|}{ Intercepts } \\
\hline 0 "very satisifed" & -0.740 & & -1.219 & & 3.104 & & 2.762 & \\
\hline 1 & -0.162 & & -0.632 & & 3.797 & & 3.461 & \\
\hline 2 & 0.208 & & -0.252 & & 4. 261 & & 3.931 & \\
\hline 3 & 0.867 & & 0.424 & & 5.094 & & 4.776 & \\
\hline 4 & 1.409 & & 0.980 & & 5.777 & & 5.469 & \\
\hline 5 & 1.999 & & 1.580 & & 6.502 & & 6.199 & \\
\hline Pseudo RSquare & 0.023 & & 0.048 & & 0.293 & & 0.302 & \\
\hline $\begin{array}{c}-2 \log \text { Lilkelihood } \\
==P<.05\end{array}$ & 19169.235 & & 19014.872 & & 18990.605 & & 18302.282 & \\
\hline$* *=P<.01$ & Note:Bold fig & ures inc & ate odds ratic & & & & & \\
\hline
\end{tabular}

Reference categories: Whites for Race, Those with at least four year of college education for Education 
Table 4. Ordinal Logit Regression Coefficients Estimating Effects of Patient Characteristics on the Level of Difficulty Getting Needed Care ( $N=5573$, Source: 2007 HTHS)

\begin{tabular}{|c|c|c|c|c|c|c|c|c|}
\hline & \multicolumn{2}{|c|}{ Model 1} & \multicolumn{2}{|c|}{ Model 2} & \multicolumn{2}{|c|}{ Model 3} & \multicolumn{2}{|c|}{ Model4 } \\
\hline & Coefficient & SE & Coefficent & SE & Coefficent & SE & Coefficent & SE \\
\hline \multicolumn{9}{|l|}{ Independent Variable } \\
\hline \multirow[t]{2}{*}{ Black } & -0.156 & 0.109 & $-0.292^{*}$ & 0.115 & -0.161 & 0.110 & $-0.295^{\circ}$ & 0.116 \\
\hline & 0.854 & & 0.747 & & 0.850 & & 0.744 & \\
\hline \multirow[t]{2}{*}{ Hispanic } & -0.082 & 0.165 & -0.041 & 0.173 & -0.077 & 0.166 & -0.062 & 0.174 \\
\hline & 0.921 & & 0.960 & & 0.921 & & 0.940 & \\
\hline \multirow[t]{2}{*}{ Others } & $0.508 \cdots$ & 0.149 & $0.417^{* *}$ & 0.159 & $0.519^{* *}$ & 0.150 & $0.432^{*}$ & 0.160 \\
\hline & 1.662 & & 1.517 & & 1.677 & & 1.539 & \\
\hline \multirow[t]{2}{*}{ Family Income } & $-0.082^{* *}$ & 0.018 & $-0.039^{*}$ & 0.019 & $-0.073^{* \cdots}$ & 0.018 & -0.033 & 0.019 \\
\hline & 0.922 & & 0.962 & & 0.930 & & 0.969 & \\
\hline \multirow[t]{2}{*}{ Less than High School } & $-0.521^{* 0}$ & 0.142 & $-0.703^{* \cdots}$ & 0.150 & $-0.571^{* \infty}$ & 0.163 & $-0.746^{\circ}$ & 0.171 \\
\hline & 0.594 & & 0.495 & & 0.541 & & 0.460 & \\
\hline \multirow[t]{2}{*}{ High School } & $-0.237^{* 0}$ & 0.094 & $-0.377^{* 0}$ & 0.100 & $-0.284^{\cdots 0}$ & 0.122 & $-0.417^{\circ}$ & 0.128 \\
\hline & 0.789 & & 0.686 & & 0.719 & & 0.634 & \\
\hline \multirow[t]{2}{*}{ Some College } & -0.095 & 0.095 & $-0.246^{*}$ & 0.101 & -0.132 & 0.113 & $-0.262^{*}$ & 0.118 \\
\hline & 0.904 & & 0.782 & & 0.876 & & 0.803 & \\
\hline \multirow[t]{2}{*}{ Language Difficulty } & & & $0.522^{* *}$ & 0.089 & & & $0.445^{\circ}$ & 0.090 \\
\hline & & & 1682 & & & & 1.553 & \\
\hline \multirow[t]{2}{*}{ Economic Hardship } & & & $1.389^{*}$ & 0.079 & & . & $1.357^{* 0}$ & 0.080 \\
\hline & & & 3.979 & & & & 3.854 & \\
\hline
\end{tabular}

Mediator

Patient Activation

$\begin{array}{ccc}-0.022 * * & 0.002 & -0.020 * * \\ 0.978 & 0.981 & 0.002\end{array}$

Control Variable

$\begin{array}{lcccccccc}\text { Age } & -0.038^{* *} & -0.038 & -0.029^{* *} & 0.003 & -0.039^{* *} & 0.002 & -0.030^{* *} & 0.003 \\ \text { Female } & 0.963 & & 0.971 & & 0.962 & & 0.970 & \\ & 0.492^{* *} & 0.492 & 0.450^{* *} & 0.078 & 0.515^{* *} & 0.075 & 0.468 * * & 0.079 \\ \text { Self-Rated Health } & 1.636 & & 1.568 & & 1.674 & & 1.590 & \\ & -0.382^{* *} & 0.382 & -0.281^{* *} & 0.036 & -0.325^{* *} & 0.035 & -0.234^{* *} & 0.037 \\ & 0.682 & & 0.757 & & 0.723 & & 0.802 & \end{array}$

Intercepts

No problem

$-1.793$

$-0.483$

$-3.121$

$-1.727$

Put Off Needed Care

$-0.485$

0.896

$-1.791$

$-0.328$

\begin{tabular}{|c|c|c|c|c|}
\hline Pseudo R Square & 0.123 & 0.203 & 0.144 & 0.218 \\
\hline $\begin{array}{c}-2 \text { Log Likeli hood } \\
==P<.05\end{array}$ & 5916.205 & 5656.786 & 6374.429 & 5816.953 \\
\hline$\because n=P<01$ & Note:Bold & te odds ratios & & \\
\hline
\end{tabular}

Reference categories: Whites for Race, Those with at least four year of college education for Education 


\section{References}

Bourdieu, Pierre. 1977. Outline of a Theory of Practice. Cambridge, United Kingdom: Cambridge University Press. [1980] 1990.

Browne, K, Roseman, D, Shaller, D, \& Edgman-Levitan S. 2010. "Measuring Patient Experience As A Strategy For Improving Primary Care." Health Affairs, $5(29): 921-925$

Bugyi, Paul. "The Role of Cultural Capital in the relationship between Socioecnomic Status and Health Outcomes: The Case of Hemodialysis Patient Compliance." Vol. 27. Socioal Sources of Disprities in Health and Health Care and Linkages to Policy, Population Concerns and Providers of Care. Ed. Jennie J. Knenfeld. Tempe,AZ: Emerald, 2009.

Casagrande, S et al. (2007). "Perceived Discrimination and Adherence to Medical Care in a Racially Integrated Community". Journal of general internal medicine, 22 (3); 389.

Chernew, M, Mechanic, R, Landon, B, \& Safran, D. 2011. "Private-payer innovation in massachusetts: the 'alternative quality contract'." Health Affairs, 1(30): 51-61.

Cleary, PD 1999. "The increasing importance of patient surveys". Quality in health care , $8(4): 212$. 
Cunningham, J, Hargraves, J, \& Hughes, J. 2001." Racial and ethnic differences in access to medical care in managed care plans." Health Service Research, 36(5): 853.

Dranove, David. 2000. The evolution of american health care: from marcus welby to managed care. Priceton : Priceton University

Elliott, M, Lehrman, W, Goldstein, E, \& Hambarsoomian, K. 2009.. "Do Hospitals Rank Differently on HCAPHS for Different Patient Subgroups?." Medical Care Research and Review, 67:56-73

Feldstein, P. 2005. Health care economics. New York: Thomson Delmar Learing.

Goldsmith, Jeff. 2011. "Accountable care organizations: the care for flexible partnerships between health plans and providers." Health Affairs, 1(30): 32-40 Goldstein, Lehrman, Elliott, Hambarsoomian, \& Giordano E 2010. "Racial ethnic differences in patients? Perceptions of inpatient care using the HCAHPS survey". Medical care research and review , 67 (1): 74 .

Hausmann, L et al. 2008. "Perceived Discrimination in Health Care and Use of Preventive Health Services". Journal of general internal medicine, 23 (10); 1679. Hasnain-Wynia, R., Baker, Nerenz.,D., Feinglass, J., Beal, C.,\& Landrum, M. 2007. "Disparities in health care are driven by where minority patients seek care: Examination of the hospital equality alliance measures." Archives of Internal Medicine, 167:101-107 
Hibbard, J, Stockard, J, Mahoney, E, \& Tusler, M. 2004. " Development of the patient activation measure (pam): conceptualizing and measuring activation in patients and consumers." Health Services Research, 4: 39.

Hibbard, J, .Mahoney, E, Stockard, J, \& Tusler, M. 2005. "Development and testing of a short form of the patient activation measure.". Health services research, 40 (6): 1918.

Hibbard, JH, Mahoney, ER, Stock, R, \& Tusler, M 2007. "Do increases in patient activation result in improved self-management behaviors?". Health services research, 42 (4): 1443.

Hibbard,J, Cunningham, P. 2008 "How Engaged Are Consumers in Their Health and Health Care, and Why Does It Matter." Heath System Change 8: 1-9.

Jha, A.K., Ovar,J., Zheng, J.,\& Epstein, A.2008. "Patients' perception of hospital care." Archives of Internal Medicine, 167:1177-1182.

Landon, B, Gill, J, Antonelli, R, \& Rich, E. 2010. "Prospects for rebuilding primary care using the patient-centered medical home." Health Affairs, 5(29): 827-834.

Lieberman, S, \& Bertko, J. 2011. "Building regulatory and operational flexibility into accountale care organizations". Health Affairs, 1(30): 23-31

Link, Bruce G., Mary E. Northridge, Jo C. Phelan, and Michael L. Ganz. 1998. "Social Epidemiology and the Fundamental Cause Concept: On the Structuring 
of Effective Cancer Screens by Socioeconomic Status."Milbank Quarterly 76:375-402.

Lubetkin, Erica, Lu, wei-Hsin, \& Gold, R Martha. 2010. "Levels and Correlates of Patient Activation in Health Center Setting: Building Strategies for Improving Health Outcomes. "Journal of Health Care for the Poor and Underserved, 21(3): 796-808.

Lutfey, Karen E. 2001. "Practitioner Assessments of Patient Compliance with Medical Treatment Regimens: An Ethnographic Study of Two Diabetes Clinics." PhD dissertation, Department of Sociology, Indiana University, Bloomington, IN.

Lynne, R, \& Marlania, N 2010. "Access to Health and Health Care: How Race and Ethnicity Matter." Mount Sinani Journal of Medicine, 77 (2): 166-177.

Morales, LS, Elliott, MN, Weech-Maldonado, R, Spritzer, \& Hays RD. 2001. "Differences in CAHPS adult survey reports and ratings by race and ethnicity: an analysis of the National CAHPS benchmarking data 1.0.". Health services research, 36 (3): 595.

Murray-García, Jann L 2000. "Racial and Ethnic Differences in a Patient Survey: Patients' Values, Ratings, and Reports regarding Physician Primary Care Performance in a Large Health Maintenance Organization". Medical care , 38 (3): 300. 
Perloff, Richard 2006. "Doctor-Patient Communication, Cultural Competence, and Minority Health". The American behavioral scientist.Beverly Hills, 49 (6): 835.

Remmers, C et al. 2009. "Is Patient Activation Associated With Future Health Outcomes and Healthcare Utilization Among Patients With Diabetes?". The Journal of ambulatory care management, 32 (4): 320.

Rodriguez, H, Scoggins, F, Von Glahn, T, Zaslavsky, M, \& Safran, D,2009. "Attributing sources of variation in patients' experiences of ambulatory care". Medical care,47 (8):835.

Shi, Leiyu (12/01/2009). "The influence of English proficiency on access to care". Ethnicity \& health (1355-7858), 14 (6), p. 625.

Shim, K. 2010." Cultural health capital: a theoretical approach to understanding health care interactions and the dynamics of unequal treatment." Journal of health and social behavior, 51(1): 1-15.

Saperstein, A. (2009). Different Measures, Different Mechanisms: A New Perspective on Racial Disparities in Health Care. In J. J. Knenfeld (Ed.), Socioal Sources of Disprities in Health and Health Care and Linkages to Policy, Population Concerns and Providers of Care, Tempe, AZ: 21-45.

Yang, J \& Singer, MK 2007. "Increasing Access to Care for Cultural and Linguistic Minorities: Ethnicity-Specific Health Care Organizations and Infrastructure". Journal of health care for the poor and underserved, 18 (3): 532. 


\section{Appendix A: Patient Activation Measurement}

Your Name:

Today's Date:

For Official Use only: MRN

\section{Me and My Health}

INSTRUCTIONS: For each statement below please mark an $\mathrm{X}$ in the box to the right that best describes how much you disagree-agree with the statement as it applies to you personally.

There are no right or wrong answers. Your answers should be what is true to you and not just what you think the doctor wants you to say.

$$
\begin{gathered}
\text { Strongly Disagree Agree } \begin{array}{c}
\text { Strongly } \\
\text { Agree }
\end{array} \\
\text { Disagree }
\end{gathered}
$$

1. When all is said and done, $I$ am the person who is responsible for managing my health condition.

2. Taking an active role in my own health care is the most important factor in determining my health and ability to function.

3. I am confident that I can take actions that will help prevent or minimize some symptoms or problems associated with my health condition.

4. I know what each of my prescribed medications do.

5. I am confident that I can tell when I need to go get medical care and when I can handle a health problem myself.

6. I am confident I can tell a doctor concerns I have even when he or she does not ask.

7. I am confident that I can follow through on medical treatments I need to do at home.

8. I understand the nature and causes of my health condition(s).

9. I know the different medical treatment options available for my health condition.

10. I have been able to maintain the lifestyle changes for my health condition that I have made.

11. I know how to prevent further problems with my health condition.

12. I am confident I can figure out solutions when new situations or problems arise with my health condition.

13. I am confident that I can maintain lifestyle changes, like diet and exercise, even during times of stress.

(PAM) 


\title{
Appendix B: Waiver for Human Subjects Research Review
}

\author{
REVIEW NOT REQUIRED \\ HUMAN SUBJECTS RESEARCH REVIEW COMMITTEE (HSRRC) \\ 122127 \\ RECEIVED \\ Office of Research and Strategic Partnerships, Portland State University \\ FEB 242012 \\ 620 Market Center Building, (503) 725-4288 / 1-887-480-4400 e-mail: hsrredtists.pdx.edu
}

It is the authority of the HSRRC, not the investigator, to determine whether or not a research project involving human subjects requires review by the HSRRC. The purpose of this form is to provide the investigator with written documentation showing that the HSRRC was consulted and deemed the project "No Review Required."

This form is most commonly used when a project involves analysis of SECONDARY DATA WITHOUT IDENTIFIERS and meets all four of the following criteria:

1. All identifying information has been removed and data cannot be linked back to individuals.

2. No contact with subjects is/was involved.

3. Data has been previously collected by another investigator.

4. Data already exists.

Note: If the project involves secondary data but does not meet all four criteria, a complete application must be submitted.

In addition to this form, please submit a one-page summary of the project. It should detail the purpose of the study, a complete description of the data set or sample, recruitment or data collection procedures/circumstances, and an explanation of why you believe the project does not require review by the HSRRC.

\begin{tabular}{|c|c|c|c|}
\hline \multicolumn{2}{|c|}{ Principal Investigator Katsuyc Oi } & \multicolumn{2}{|l|}{ Dept Sociology } \\
\hline PSU ID No. & 975319588 & Date of Application & $02 / 24 / 2012$ \\
\hline \multirow[t]{2}{*}{ Mailing Address } & 901 Ser Kingas $\$ 511$ Porland OR & \multirow{3}{*}{$\begin{array}{l}\text { Campus Extension } \\
\text { Home/Work Tel. }\end{array}$} & \\
\hline & 97205 & & $503.3 \times 2.7753$ \\
\hline Email Address & Di.kntesaya Ggast.tom. & & \\
\hline \multicolumn{4}{|c|}{ Title of Proposed Study } \\
\hline \multicolumn{2}{|c|}{ Anticipated Length of Project } & Start Date $12 / 2+12$ & ond Patent E. \\
\hline
\end{tabular}

INVESTIGATOR'S ASSURANCE

I do hereby certify that my research as referenced above does not involve any human subjects, and/or it involves only secondary data from which all identifying information has been removed. Therefore, no review is required from the HSRRC.

$$
\text { 大年武 }
$$

Signature of Principal Investigator
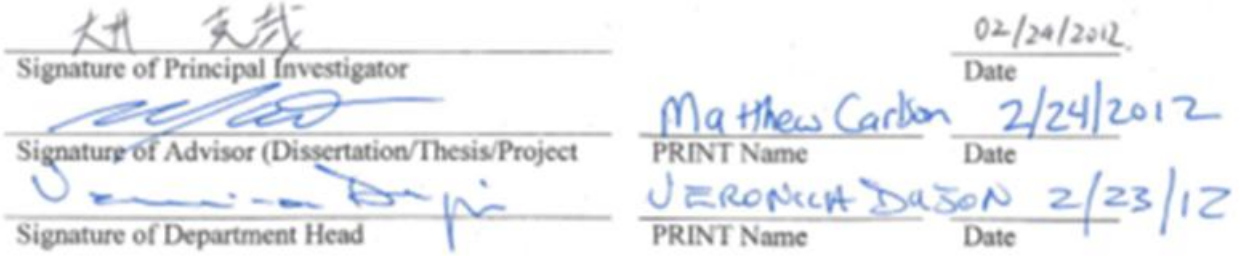

Check one: Masters Thesis Project to $\begin{aligned} & \text { Doctoral Dissertation } \\ & \text { Proposal Approval Date }\end{aligned} \quad$ Special Project $\square$

For HSRRC Only: Approved tome Suluy Date $4 / 20 / 12$ 